\title{
The Applied Research on the Optimization of Distribution Network Topological Structure Configuration Model
}

\author{
Zucheng Huang, Wei Chen, Lixia Chen and Hao Chen \\ Guangzhou Power Supply Co. Ltd. Guangzhou, Guangdong, China, 510000 \\ 2397615891@qq.com
}

\begin{abstract}
With the rapid growth of the economy and the increasing improvement of life, the large electricity consumption gradually intensifies power grid transmission pressure. In order to solve this problem, the paper analyses the trend of tremendous development in computer, electronic and electric power technology and makes a deep related research. Based on the further analysis of key technologies, the author sets constraint conditions, analyzes the supporting effect, and establishes an optimal configuration model with multiple purposes for distribution network topological structure. On the basis of the minimal module ideal point algorithm, the overall planning is conducted through deep consideration to assure the further optimal configuration of the model. Then the value will be calculated by using the POS algorithm. At last, four optimization plans are obtained and the fourth one whose weight value can be adjusted is chosen to achieve automation and the more rationalized and optimal development of the distribution network topological structure. In addition, this optimal plan provides a stronger basis of theory to the practical application of related work and industries.
\end{abstract}

Keywords: distribution network topological structure, the optimal configuration model with multiple purposes, minimal module ideal point algorithm, POS algorithm

\section{Introduction}

As the electric power and electronic technologies develop fast, the distribution network topological structure has become a leader technically and economically in many fields, enjoying a promising prospect. Its special feature of flexibility and controllability [1] mainly focuses on the fault location of DG optimization plan, voltage management, active management, demand side management (DSM) and security and protection. The characteristics of the control lie in the indirect energy consumption, DG adjustment, DG protection and DG monitoring. The core values of the distribution network topological structure are mainly the initiative of many controllable resources and distributed power management, the function of electric power distribution, the coordination of two-way electric current movement, the realization of a large quantity of distributed power access and a pretty high asset utilization rate. At present, its special feature of flexibility and controllability is used mostly in the electronic technology of the distribution network topological structure, even in the products like the air conditioner, refrigerator and washing machine. However, the traditional energy has been used greatly, and the environmental pollution arises along with the severe consumption. Unfortunately, because of a lack of measurement methods [2] and its sizes and features complying with the heterogeneous multi-source big data, the distribution network topological structure can only get the data from the historical databases and other estimated load curves. Therefore, the data error is bigger and it's more difficult to estimate the condition and the trends of the distribution network topological structure. Consequently, the traditional plan of the distribution network topological structure and its function model cannot achieve the access of high permeability and distributed energy. In this situation, a secure, 
energy-saving and energy-renewing environment where every kind of resources can be used reasonably and effectively has been an irresistible trend, so that the resource pressure in China can be alleviated and the severe nature degradation situation can be improved. ${ }^{[3]}$ Then it is necessary to establish an environmental friendly power transmission line which can reduce the consumption, increase the resource utilization and equipment efficiency with a low cost and loss but a high reliability. Moreover, the controllability, objectivity and the optimal transmission capacity of the distribution network topological structure need to be improved. The assets' security level of the distribution network topological structure should be elevated and a high permeability energy access demand can be realized. ${ }^{[4]}$ As a result, the energy area can reach an optimal configuration and a high efficiency; the range of the distribution network topological structure will be broader and the applied extent more intelligent, the users larger. The paper aims to make full use of much data to support the research and development of business and operation models.

\section{The Basic Information of the Distribution Network Topological Structure}

\subsection{The basic features}

In the era of information technology, there is much big data with a large quantity and complex layers and structures. [5] In the precondition of so much data, the integrated research of data collection, management and application, storage, search and sharing cannot be achieved in a certain period. Besides, the development of distribution network topological structure faces double challenges including environment pressure and energy shortage, especially in this urgent transformation from electric power industry into low-carbon industry. So the development of the distribution network has a significant meaning for the development of low carbon economy. In spite of the feature of big data, the distribution network features in a high capacity, speed ability, diversity and a low value density. Based on the practical application of energy-saving distribution network and the related standards and norms and taking the plan, equipment parameter and operation into account, the main static, dynamic and attrition indicators are obtained including: medium voltage distribution network, distribution transformer and low voltage area. The detailed indicators are the substation construction standard, clearly average power supply radius, precisely average cross section of trunk lines, new energy-saving conductor, economical operation transmission lines, the qualification rate of line power factor, the capacity proportion of energy storage device, distribution attrition rate, and so on. Hence, it is necessary to lay a foundation of big data technology in the application of smart power grids. [6]

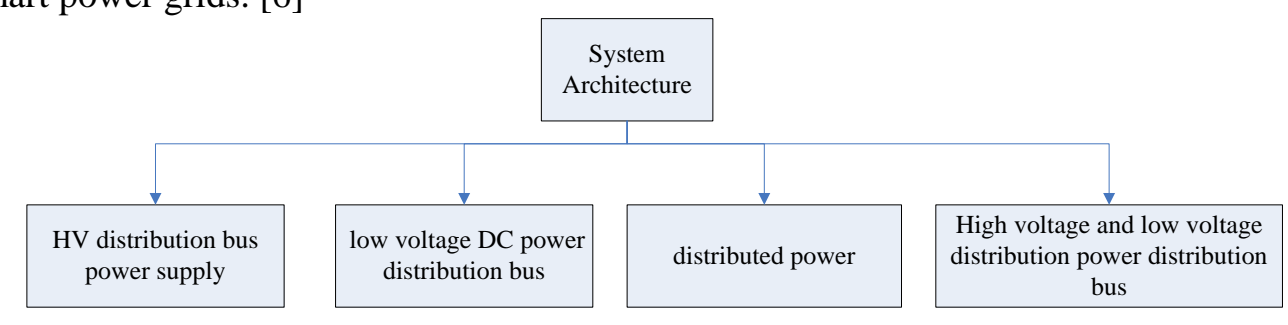

Figure 1. System Architecture Diagram

\subsection{The Construction}

Because of its randomness and intermittency, the control difficulty of optimizing distribution network increases to some extent. So the energy transmission work of optimizing the distribution network is of vital significance for the increase of equipment efficiency, the realization of high-quality power supply, the delay of capacity building, the 
high permeability of renewable energy and the increase of existing resource using rate. [7] The need of the distribution network is important, so is its construction. Now the construction content of the distribution network topological structure is concluded as follows:

Table 1 .Distribution Network Construction Content

\begin{tabular}{|c|c|}
\hline $\begin{array}{l}\text { System planning and distribution power } \\
\text { grids }\end{array}$ & System Planning \\
\hline \multirow{3}{*}{$\begin{array}{l}\text { AC-DC hybrid switching station } \\
\text { construction }\end{array}$} & $\begin{array}{l}\text { Flexible access smart micro grid } \\
\text { distribution network to meet the active } \\
\text { control and optimization }\end{array}$ \\
\hline & $\begin{array}{l}\text { Constructed hybrid AC-DC switching } \\
\text { station }\end{array}$ \\
\hline & $\begin{array}{l}\text { Construction grid, to achieve power } \\
\text { optimization flow partition, load balancing } \\
\text { and comprehensive application equipment } \\
\text { utilization }\end{array}$ \\
\hline \multirow[t]{4}{*}{ Smart Micro Grid Group Access } & $\begin{array}{l}\text { The construction of intelligent micro-grid } \\
\text { group }\end{array}$ \\
\hline & $\begin{array}{l}\text { Multi-component system energy } \\
\text { utilization ,satisfy the self-adjusting and } \\
\text { internal power exchange }\end{array}$ \\
\hline & $\begin{array}{l}\text { Positive interaction, cooperation and } \\
\text { win-win }\end{array}$ \\
\hline & $\begin{array}{l}\text { Comprehensive optimization, improve the } \\
\text { absorptive power }\end{array}$ \\
\hline \multirow{2}{*}{$\begin{array}{l}\text { Rooftop solar thermal power generation } \\
\text { access }\end{array}$} & Access Active Distribution Network \\
\hline & $\begin{array}{l}\text { By protection, monitoring and control, } \\
\text { metering and communication, distribution } \\
\text { network control and global optimization }\end{array}$ \\
\hline \multirow[t]{2}{*}{ Construction of multivariate data collection } & $\begin{array}{l}\text { Intelligent power distribution units for data } \\
\text { collection terminal }\end{array}$ \\
\hline & Provide for public service center platform \\
\hline
\end{tabular}

\subsection{Key Technologies Research}

At present, the key technologies mainly include advanced electric power and electronic, communication and information, storage, smart power and virtual power plants technologies. Next is the detailed introduction ${ }^{[8]}$ :

(1) Networking technology

The networking technology includes the flexible networking technology, energy router, plug-and-play standard interface and smart power distribution terminal unit technology. The flexible networking technology uses the self strengths of many network structure forms to achieve a broad connectivity of future distribution network topological structure. The energy router utilizes self-test, self-diagnosis and self-protection technology to improve the security performance of equipment and increase the connectivity capacity. The plug-and-play standard interface on the basis of mutual openness and share of data and electric power connects the information and physical content in virtue of itself description ability to support the automotive identification function and online automotive management of the distribution network topological structure ${ }^{[9]}$.

(2) Comprehensive sensing technology at unit level

The comprehensive sensing technology at unit level includes smart sensor network 
technology and smart power distribution terminal unit technology. The network technology is a smart function that makes information turn into data through its features of reliability, connectivity and comprehensiveness so as to achieve local convergence and analysis. The unit technology through the synchronous measurement technology collects and uploads smart network measurement information. Furthermore, it adopts flow control and power supply control methods to realize overall and local collaborative optimization of operation control.

(3) Operation and control technology

The Operation and control technology includes network topology flexible control, the flexible flow control, voltage sag governance, system energy access voltage control, three phase unbalanced governance and renewable energy high permeability zone harmonic governance technologies. All these technologies not only can achieve self-healing topology control and the flexible control of the flow direction, but also can solve the problems like voltage sag and power outages in a short time.

(4)Protection technology

The Protection technology mainly includes AC-DC hybrid distribution network topological structure protection, self-adaptation protection and network protection. Through comprehensive technologies, the DC lines can be positioned fast and accurately, the fault can be isolated and the fault area can be identified exactly.

(5) Storage technology

So far the storage technology is realized by the support of lithium battery. Through the new typed battery component technology, the charge and discharge technology of the storage system with multiple optimization purposes can be achieved to support the efficient and optimal operation.

\subsection{The Protection system}

The existing power in the grid is distributed. For a fault circuit, the direction and amplitude can change relatively, so that the grid protection can make error or mistake direction. At present, the generally basic solutions are directional over-current protection, circuit longitudinal direction protection and bulbar protection. The detailed introduction is as follows:

(1) The directional over-current protection ${ }^{[10]}$. It mostly occurs in traditional and radial distribution network topological structure. Especially, in the current situation of many power accesses, this protection is achieved through voltage transformer.

(2) The circuit longitudinal direction protection. Owing to the direction mistakes of distributed powers and the restriction of electronic devices, the flexibility of the distribution network topological structure's protection system is yet to be high. In order to increase the protection speed of direction device and the synchronous demand, the research is done according to the fault components in lines, namely, when the direction is positive, the signal is allowed to send at the same time the protection signal can be tested.

(3) The bulbar protection. It means all the lines were provided longitudinal protection. It can identify all fault directions and realize the protection devices accessible to all lines.

The characteristics of the distribution network topological structure's three protections are with a high flexibility, a quick response and an increased capacity of the distribution network.

\subsection{The Foreign Development}

The distribution network topological structure technology represents the trend of future distribution network development. Currently, scholars home and abroad are conducting related applied research actively.

In Asia: in 2004, Tokyo Institute of Technology in Japan proposed the conception of distribution system based on Micro DC-Grid and the Dc power distribution system 
prototype was made ${ }^{[11]}$. In Japan, the advanced distribution automation system has covered many cities like Yokohama, Kitakyushu and Kansai. They attach the importance to the full use of renewable resources. The main construction projects on multiple-energy are Aichi, Kyoto, Hachinohe, Kyotango, Shimizu and Sendai. In addition, the smart micro-grid was established in Myongji University, Korea.

In Europe: in 2004, on the basis of the distributed Dc power distribution system structure, Polytechnic University of Milan in Europe proposed that using photovolt and wind power to generate electricity. The members of EU including Denmark, Spain and Britain carried out the related research such as the active power distribution of supply network technology and demonstration project construction. The technology of ADINE is very influential. This technology can support the management of the distribution network topological structure and stimulate a large quantity distribution nodes accesses in the practical environment at the same time. As a result, the interactive function and the united tests of protecting voltage, control voltage and the power quality assurance can be realized. It is worth to mention that in 2003, the proposition of low carbon by Britain further promoted the development and application of the distribution network topological structure in foreign and domestic environments.

\subsection{The Domestic Development}

Our country ${ }^{[12]}$ deep realized that the optimal plan of the distribution network topological structure is vital for the secure operation of economy. Therefore, the State Grid Corporation initiates the construction of smart grid to increase the ability of distribution network energy transmission and optimization and make full use of the energy. Our country makes the society as main area, the market as the goal, the overall development as foundation to establish "Energy Internet" in demonstration cities. In 2012, the 863 plan was carried out. A deep research named "the research and application of the technology of the active distribution network's intermittent energy consumption and optimization" was conducted in the distribution network industry and other related industries. Take Beijing as an example. Beijing has established new and renewable energy demonstration counties and formed a fundamental industry cluster such as power generation industry using wind, solar, photovolt and biomass and smart grid. The multiple energy coordinated access and full consumption with flexible topology and controllable flow is achieved. In order to solve this situation, the technology of the distribution network topological structure aims at elevating the new energy utilization rate and optimizing the primary energy.

\section{The Establishment and Solving of the Optimal Model of the Distribution Network Topological Structure with Multiple Purposes}

\subsection{The Constraint Conditions}

(1) According to the fact that the battery energy storage system produced by current manufactures ${ }^{[13]}$ mostly are modularized design and package, the paper proposed that the capacity value of the energy storage system has discreteness.

(2) Considering the distribution network' s full consumption of the intermittent energy, the total value of the storage capacity is equal or larger than the minus between the total capacity of the intermittent energy and the minimum load value.

(3) The unit capacity value of the nodes configuration cannot be larger than the allowed values.

(4) The research of this paper has fully considered the previous construction and installation costs of the distribution network topological structure system. 


\subsection{The Supporting Effect}

First of all, the paper has fully considered the supporting effect of the distribution network topological structure and made a detailed analysis of the storage system's supply and storage performance to lay a solid foundation for the next part, the establishment and solving of the optimal model of the distribution network topological structure with multiple purposes.

(1) Provided that there is an ideal peak clipping energy equation, that is

$$
W=\sum_{k}\left(F_{k}-F_{j}\right) \Delta P
$$

In this equation, $\Delta P$ stands for the intervals in the load curve.

From the foregoing equation, the total power input of the energy storage system can be defined as follows:

When time $T$ feeder load is larger than the average load, the system in that time can have a discharge phenomenon. In the equation, $L$ stands for the total energy of the energy storage system, and the discharge power is as follows:

$$
F_{\alpha}= \begin{cases}\left(F_{1}-F_{2}\right) \frac{L}{W} & L \leq W \\ F_{1}-F_{2} & W>L\end{cases}
$$

(2)

When time $T$ feeder load is smaller than the average load, the system in that time can have a charge phenomenon. The charge power is as follows:

$$
F_{\alpha}= \begin{cases}\left(F_{2}-F_{1}\right) \frac{L}{W} & L \leq W \\ F_{2}-F_{1} & W>L\end{cases}
$$

Combine the equation (1) and (2), and a new one can be obtained as follows:

$$
F_{\alpha}^{\prime}=\left\{\begin{array}{cc}
\left(1-\frac{L}{W}\right) F & L \leq W \\
0 & W>L
\end{array}\right.
$$

(4)

Through the summary, the improvement extent achieved is as follows:

$$
\Delta F_{\alpha}=F-F^{\prime}=\left\{\begin{array}{cc}
\left(\frac{L}{W}\right) F & L \leq W \\
0 & W>L
\end{array}\right.
$$

The conclusion of the above research is that the system' s peak-shift role can be obviously and gradually improved as the total system energy increases. When the ideal peak clipping energy is achieved, the conclusion that the peak valley equals to zero can be obtained.

(1) The increase of voltage quality

Considering the voltage quality, the improved model of voltage indicators can be obtained, in which $V_{\max }, V_{\min }$ stand for the top and bottom limitations respectively, F stands for power, $\mathrm{K}$ stands for the number of nodes:

$$
I_{k}=\frac{\left(D_{i}-D_{\text {min }}\right)\left(D_{M A X}-D_{i}\right)\left|F_{i}\right|}{\left(D_{n}-D_{\text {min }}\right)\left(D_{\max }-D_{n}\right) \sum\left[F_{j}\right.}
$$




$$
I=\sum_{i=1}^{k} I_{k}
$$

The above research makes a conclusion: when the voltage is smaller than the bottom limitation, the voltage amplitude of every node increases through the power sending out and the voltage is much nearer the rated value of the node voltage; while the voltage is larger than the top limitation, the voltage amplitude decreases through the power absorption and the power value increases.

(2) The adjustment of electric power capacity

After the full consideration of situations that there are constraints between the inequations and the node voltage inequality, the paper obtains the maximum positive regulation force $\mathrm{F}$ of the distribution network topological structure as follows:

$$
\left\{\begin{array}{c}
F=\mathrm{max} \sum_{i=1}^{k} F \\
\text { S.T. } F_{i} \leq F_{\max } \\
g\left(F_{\max }\right) \leq 0
\end{array}\right.
$$

\subsection{Model Establishment and Solving}

Besides the constraint conditions change, the optimal model of the distribution network topological structure with multiple purposes changes a lot in the controlled variable and objective function.

(1) Model assumption

In the motion of particle swarm, because the capacity off-limit particles have a little influence, they are not considered in terms of the overall optimization solving and optimal individual.

(2) Model establishment

The optimal model of the distribution network topological structure with multiple purposes is established on the basis of consideration of load shifting, the comprehensive quality of voltage and the initiative of power adjustment. In this model, $\int_{1}$ stands for the objective value of achieving load shifting, $\int_{2}$ stands for the computing situation of comprehensive operational conditions, $\int_{3}$ the objective value of maximum positive adjustment capacity, $\int_{4}$ the objective value of maximum negative adjustment capacity.

$$
\max W=\max \left(\int_{1}, \int_{2}, \int_{3}, \int_{4}\right)
$$

According to the practice, the larger the total energy value of the energy storage system is, the more obvious the decrease of off-peak difference is. To maximize the interest, the above equation is changed into the constraint conditions and the optimal model of the distribution network topological structure with multiple purposes is obtained as follows:

$$
\left\{\begin{array}{c}
\max =\operatorname{ma}\left(\int_{2}, \int_{3}, J_{4}\right) \\
\text { S.T. } y_{i}=n L_{0} \quad i \in[1, n) \\
y_{i} \leq P^{\prime} \\
\sum_{i=1}^{n} y_{i} \geq F-F^{\prime} \\
\sum_{i=1}^{n} y_{i}=F_{\mathrm{max}} \\
g\left(x_{i}\right)=0
\end{array}\right.
$$


In this paper, the $\mathrm{g}\left(x_{i}\right)=0$ stands for the equation constrain of power balance and $\mathrm{n}$ stands for integers.

(3) Model solving

The flow chart of the model solving is as follows:

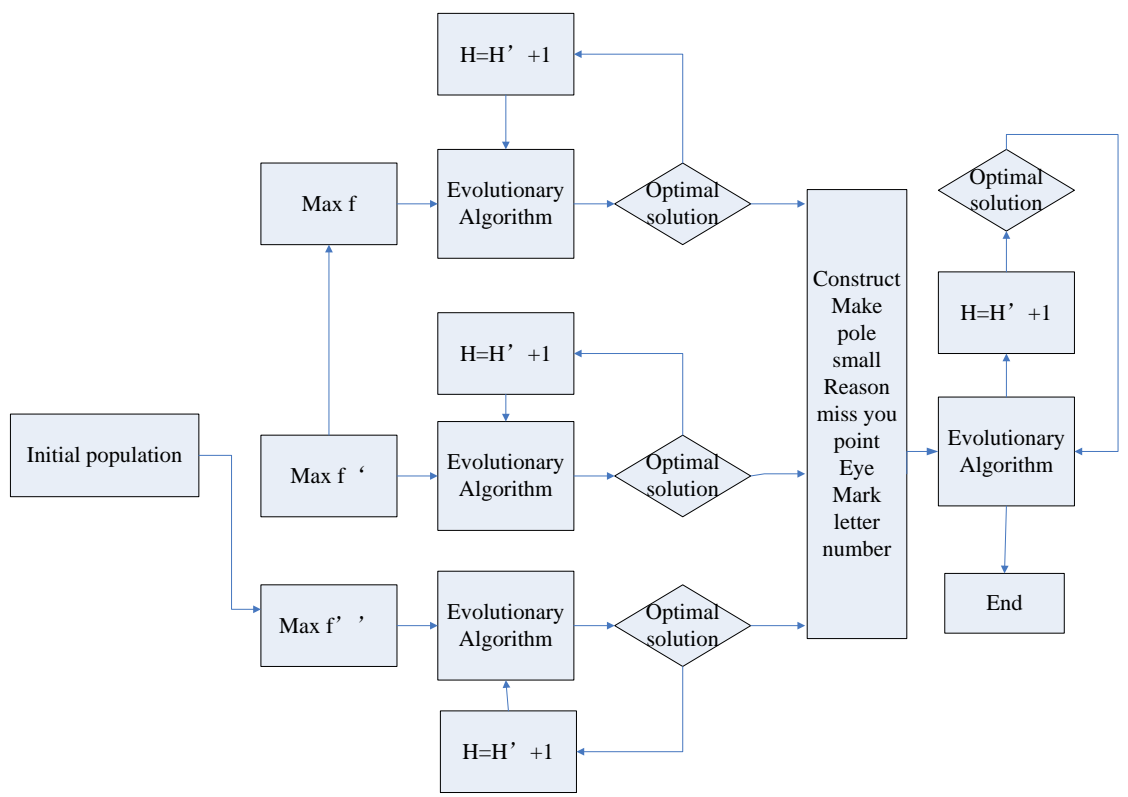

Figure 2. Work Process

Through the minimal module ideal point algorithm [14], the single objective function with several purposes combination is as follows:

$$
\min \int=\lambda_{2}\left|\frac{\int_{2}-\int_{2}^{*}}{\int_{2}^{*}}\right|+\lambda_{3}\left|\frac{\int_{3}-\int_{3}^{*}}{\int_{3}^{*}}\right|+\lambda_{4}\left|\frac{\int_{4}-\int_{4}^{*}}{\int_{4}^{*}}\right|
$$

The above equation can reflect the quality of the solution and obtain the optimal solution that can be adjusted according to the practice. In the equation, the ideal point' $s$ reflection is $*$, the weight values $\lambda_{2}, \lambda_{3}, \lambda_{4}$ stand for the objectives $\int_{2}, \int_{, 3}, \int_{4}$ respectively. Every sub-weight values $\lambda_{2}^{*}, \lambda_{3}^{*}, \lambda_{4}^{*}$ stand for the deviation between their objective values and the ideal objective values. At the same time, the weight values have the relationship as follows:

$$
1=\lambda_{2}+\lambda_{3}+\lambda_{4}
$$

In order to further solve the issues of consistent and unified dimension of the distribution network topological structure' $s$ multiple purpose function, the paper makes an overall plan and ensures the optimal configuration, so that the equation is improved as follows:

$$
\begin{aligned}
& e_{i}^{n+1}=e_{i}^{n}+R_{0} \int_{l}\left(c_{i}^{n}\right) \quad c_{i}^{n} \geq 0 \\
& e_{i}^{n+1}=e_{i}^{n}+R_{0} \int_{l}\left(-c_{i}^{n}\right) \quad c_{i}^{n}<0
\end{aligned}
$$

Through the POS algorithm, the particle swarm is iterated on the basis of the entire particles to change the whole vectors. A configuration plan is obtained as follows: 
Table 2. Configuration Tables

\begin{tabular}{ccccccc}
\hline Numb & $\begin{array}{c}\text { Weight } \\
\text { setting }\end{array}$ & Weights & index & $\begin{array}{c}\text { Positive } \\
\text { regulation }\end{array}$ & $\begin{array}{c}\text { Negative } \\
\text { regulation }\end{array}$ \\
\cline { 1 - 2 } 1 & N / A & & $\begin{array}{c}\text { Non-configured energy storage } \\
\text { system }\end{array}$ & 0.144 & 0 & 0 \\
2 & $1 / 0 / 0$ & $\left(Q_{5}, 5\right) /\left(Q_{14}, 5\right) /\left(Q_{16}, 5\right) /\left(Q_{18}, 5\right) /\left(Q_{21}, 5\right)$ & 0.987 & 2.514 & 1.200 \\
3 & $0 / 1 / 0$ & $\left(Q_{4}, 5\right) /\left(Q_{10}, 5\right) /\left(Q_{12}, 5\right) /\left(Q_{18}, 5\right) /\left(Q_{19}, 5\right)$ & 0.991 & 2.564 & 1.594 \\
4 & $0.1 / 0.3 / 0.6$ & $\left(Q_{1}, 5\right) /\left(Q_{2}, 5\right) /\left(Q_{6}, 5\right) /\left(Q_{14}, 5\right) /\left(Q_{16}, 5\right) /\left(Q_{5}, 5\right)$ & 0.764 & 2.894 & 1.984 \\
\hline
\end{tabular}

From Table 2 we can conclude that, the Plan 1 doesn' t consider the energy storage system so that it is not practical; the Plan 2 would realize the optimal configuration of the power positive adjustment capacity if it installed the energy storage system near the maximum load points; the Plan 3, would realize the optimal configuration of the power negative adjustment capacity if it installed the energy storage system near the nodes; in Plan 4, the optimal configuration would be realized and the optimal voltage would be ensured if the weight values were adjusted to complete the initiative optimization. In a word, the Plan 4 is the most reasonable applied plan which simplifies the method of solving the model, reduces the complexity of variables and ensures the efficiency and accuracy of the model. This plan has solved the related problems and obtains more accurate results.

\section{Conclusion}

The development of the distribution network topological structure directly affects the production and life of people, especially in this era with rapid technological development and complex and changeable situation. It also has a strong influence on the development of the electricity transmission cause. Through the deep research on the related content and technologies of the distribution network topological structure, the paper establishes a model, proposes an automotive optimization plan of it by regulating the weight value according to the practice and ensuring the quality of the electricity. The proposition of this plan not only promotes the development of the distribution network topological structure cause but also affects a lot related industries such as the electric power and the machinery. It provides a theoretic foundation and propels the development of the whole electric network.

\section{Development Prospect}

With the rapid growth of the economy and the increasing improvement of life, users have higher demand on the electricity quality and electric power capacity. Therefore, the development of the distribution network attracts much attention of scholars and experts with a vital and promising prospect. The research includes the multi-state research, the multi-environment research, and the smart grid research and so on.

(1) Multi-state research

The consideration of the distribution network topological structure module need to consider its states including three conditions: the state of wind power generation, the state of photovoltaic power generation and the multi-state load based on the time sequence. Through the model establishment under the different confidence levels, the plan with a higher economic profit can be obtained based on the constraint conditions so that it can satisfy the practical need and achieve the goal and the significance.

(2) Multi-environment research

By fully considering two comprehensive optimization configurations of the distribution network topological structure: the active resource and the reactive resource, we need to 
find an applied plan with the optimal resource utilization rate, the maximum economic profit and the controllability in different times so as to ensure the time sequence and randomness of the accurate computation. The different applied situations are taken into account under the different confidence levels to provide a theoretic foundation and reference value for the plan of the distribution network topological structure in the same industry and related industries.

(3) Smart research

The development of smart the distribution network topological structure in the future is the main trend. The experiment in this paper mainly conducts the data simulation which exchanges through the initiative distribution of the distributed power to achieve the protection and voltage controls. Through the wide connectivity of the future grid, the distribution network topological structure can make the information circulation and share into reality, and become diverse, mutually supported and flexible. It can exert the high intelligence of the grid by coordinating the centralized dispatching and scattering the self-optimization to realize the fault self-recovery in the process of network rebuilding and provide secure power.

(4) User research

The promising technologies in the future are mainly the recycled electric car, the energy storage utilization, the user interaction and the conception of VMG. In these technologies, the users' application is the most important one. The openness of the distribution network topological structure should be used to achieve information share and make it accessible to the whole society and become multi-win. The distribution network topological structure shoulders the responsibility and obligation of power transmission. As the large quantity of powers access, the electricity current of the distribution network topological structure now is transforming from one-way transmission into two-way transmission.

(5) Low-carbon research

The development of low carbon electric power system is the priority in the modern society. The utilization of the renewable energy needs to master the exploitation and utilization of energy with a long distance, a large capacity and a low loss. Furthermore, the links of distribution network topological structure can achieve the need for the renewable energy generation and the supply system between the terminals. The most important is that it can mainly solve the problem of time disunity corresponding to different low carbon retardation on the basis of technologies, management and market mechanism.

\section{References}

[1] Y. M. Atwa and E. F. El- Saadany, "Reliability evaluation for distribution system with renewable distributed generation during islanded mode of operation", IEEE Transactions on Power Systems, vol. 2, no. 24, (2009), pp. 572- 581 .

[2] S. H. Karaki, R. B. Chedid and R. Ramadan, "Probabilistic performance assessment of autonomous solar-wind energy conversion systems", IEEE Transactions on Energy Conversion, vol. 14, no. 3, (1999), pp. $766-772$.

[3] J. P. Wu and C. L. Jaeseok, "A probabilistic reliability evaluation of power system including solar/photovoltaic cell generator", Power and Energy Society General Meeting. Calgary: Power and Energy Society General Meeting, (2009).

[4] S. C. Lago-Gonzalez, "A Reliability modeling of generation systems including unconventional energy sources", IEEE Transactions on Power Apparatus and Systems, vol. 5, no. 104, (1985), pp. 1049- 1056.

[5] M. Adrian, "Big data: it's going mainstream and it's your next opportunity", Teradata Magazine, vol. 5, no. 1, (2011), pp. 3-5.

[6] P. B. Kenneth, G. Lakshmi and R. Robbert van, "Running smart grid control software on cloud computing architectures", Workshop on Computational Needs for the Next Generation Electric Grid. Ithaca: Lawrence Berkeley National Laboratory, (2011).

[7] T. Shun and X. Xiangning, "Infrastructure of the power quality assessment system of power systems", Transactions of China Electrotechnical Society, vol. 25, no. 4, (2010), pp. 171-175(in Chinese). 
[8] C. D. Xu and K. W. E. Cheng, "A survey of distributed power system- AC versus DC distributed power system", 4th International Conference on Power Electronics Systems and Applications, IEEE, (2011); Hong Kong, China.

[9] H. Kakigano, Y. Miura and T. Ise, "Low-voltage bipolar-type DC microgrid for super high quality distribution", IEEE Transactions on Power Electronics, vol. 25, no. 12, (2010), pp. 3066- 3075.

[10] J. Jin, J. Allan, C. J. Goodman and K. Payne, "Single pole-to-earth detection and location on a fourth-rail DC railway system”, IEE Proceedings Electric Power Applications, (2004).

[11] Y. You, D. Liu and W.-P. Yu, "Technology and its trends of active distribution network", Automation of Electric Power Systems, vol. 36, no. 18, (2012), pp. 10-16.

[12] Z. Ji, J.-R. Zhou and W.-L. Liao, "A novel intelligent single particle optimizer", Chinese Journal of Computers, vol. 33, no. 3, (2010), pp. 556- 561.

[13] J.-H. Wang, N.-L. Tai and K. Song, "Penetration level permission of for DG in distributed network considering relay protection", Proceedings of the CSEE, (2010).

[14] P. E. Labis, R. G. Visande and R. C. Pallugna, "The contribution of renewable distributed generation in mitigating carbon dioxide emissions", Renewable and Sustainable Energy Reviews, vol. 15, no. 9, (2011), pp. 4891-4896.
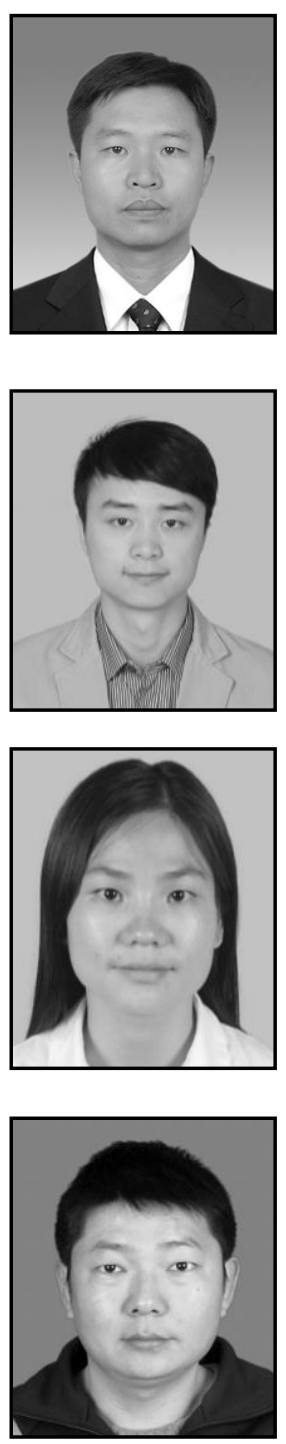

\section{Authors}

Zucheng Huang. Zucheng Huang was born in 1973, in Haifeng, Guangdong. In 2006, He graduated in South China University of Technology with a Master Degree of Power System and Automation. His research fields are intelligent distribution network key technology and operational management. His professional title is senior engineer.

Wei Chen. Wei Chen was born in 1985, in Huanggang, Hubei. In 2012, He graduated in China Three Gorges University with a Master Degree of Power System and Automation. His research fields are intelligent distribution network operation and automation. His professional title is engineer.

Lixia Chen. Lixia Chen was born in 1989, in Jieyang, Guangdong. In 2012, she graduated in South China University of Technology with an undergraduate Degree of Power System and Automation. Her research fields are intelligent distribution network operation and automation technology. Her professional title is engineer assistant.

Hao Chen. Hao Chen was born in 1983, in Huanggang, Hubei. In 2006, He graduated in Henan Polytechnic University with an undergraduate Degree of Electronic Information Technology. His research fields are the grid security and monitoring technology. 
International Journal of Grid and Distributed Computing Vol. 9, No. 12 (2016) 